\title{
Cytogenetic and molecular studies of trisomy 13
}

\author{
TERRY HASSOLD*, PATRICIA A JACOBS*, MARK LEPPERT + , AND \\ MICHAEL SHELDON* \\ From * the Division of Human Genetics, Department of Pediatrics, Cornell University Medical College, New \\ York, NY 10021; and the Howard Hughes Medical Institute, University of Utah Medical Center, Salt Lake \\ City, Utah 84132, USA.
}

SUMmaRY Chromosome heteromorphisms, restriction fragment length polymorphisms, or both were used to study the parental origin of 33 cases of simple trisomy 13 and eight cases of translocation trisomy 13 . The most common origin for the simple trisomies was non-disjunction at maternal meiosis I, while for the translocations an equal number of paternally and maternally derived cases was observed.

In seven of the simple trisomies, information was obtained from both the cytogenetic and molecular markers, making it possible to study recombination between the two non-disjoined chromosomes. Five of the seven cases involved errors at meiosis I, with crossing over being detected in two of three cases of maternal origin and in one of two cases of paternal origin. This indicates that absence of recombination because of pairing failure is unlikely to be of major importance in the genesis of trisomy 13.

Almost all information on the parental origin of autosomal trisomy has come from analysis of chromosome heteromorphisms, cytologically detectable variants which occur regularly on 10 of the 22 autosomes. This technique has been most extensively used to investigate the origin of trisomy 21 in liveborns and data are now available on over 1000 families. ${ }^{12}$ Additionally, there are limited data on liveborn subjects with trisomy $13^{3}$ and on spontaneously aborted conceptuses with trisomies 3 , $4,9,13,14,15,16,21$, and $22 .^{4}$

Cytogenetic heteromorphisms have provided useful information on the relative importance of different meiotic errors to the genesis of trisomy, but there are several important limitations to the technique. For example, the $\mathrm{X}$ chromosome and the majority of the autosomes are not heteromorphic and therefore cannot be studied with this method, and there is relatively little variation in the heteromorphic regions of several other chromosomes (for example, 3, 4, 9, and 16). Furthermore, analysis of the variants involves subjective evaluation of size and staining intensity and, even when the scoring is done blindly and by independent observers, it is possible that a small proportion of parental origin assignments will be wrong.

Some of these difficulties can now be overcome by applying restriction fragment length polymorphism

Received for publication 3 April 1987.

Accepted for publication 21 April 1987.
(RFLP) analysis to the study of the origin of trisomy. The advantages of this approach are that (1) the detection of allelic variation using RFLPs is objective; (2) the DNA fragments show gene dosage, a prerequisite for determination of the parental origin of trisomies using a two allele system, and (3) the availability of multiple polymorphisms on each chromosome makes it likely that the parental origin of virtually every trisomy can be unambiguously determined. Preliminary results from studies that have used RFLPs clearly show the efficiency of this method. ${ }^{5}$

Additionally, by combining analysis of chromosome heteromorphisms with analysis of RFLPs, it is now possible to address questions which were previously intractable. One of the most interesting of these is the possibility that aberrant levels of recombination may be important in non-disjunction. In species with chiasmate meiosis, maintenance of pairing in meiosis I is presumably dependent on the presence of at least one chiasma per bivalent. ${ }^{6}$ Thus, reduction or elimination of crossing over may lead to univalent formation and ultimately to nondisjunction and there is some evidence from studies of experimental animals ${ }^{78}$ to suggest that this may be an important source of trisomy. Furthermore, if multiple exchanges impede the orderly separation of bivalents at anaphase I, abnormally high levels of recombination might also be associated with nondisjunction. 
Both of these suggestions can now be directly tested in humans by using centromere mapping techniques to compare the frequency of crossing over in normal meioses and meioses resulting in trisomy. The mapping method using trisomy is essentially the same as that previously described for ovarian teratomas, ${ }^{9}$ that is, chromosome heteromorphisms or pericentromeric RFLPs or both are used to determine the meiotic stage of origin of the additional chromosome and centromeric distances of RFLPs estimated by evaluating the level of heterozygosity among trisomic RFLPs for which the parent of origin is known to be heterozygous. In trisomy of meiosis I origin, increasing homozygosity is directly proportional to increasing centromeric distance; in trisomy of meiosis II origin the opposite relationship holds.

Using this approach, Antonarakis et al ${ }^{10}$ recently studied the inheritance of RFLPs in 34 subjects with Down's syndrome, their parents, and normal sibs. They observed statistically significant reductions in the map distances associated with trisomy 21 and were only rarely able to detect recombination involving the two non-disjoined chromosomes. From this they suggested that defective pairing may be responsible for a large proportion of cases of trisomy 21 .

In this report, we present our preliminary observations on centromere mapping in trisomy 13 , and the results of our cytogenetic and molecular studies of parental origin in 33 cases of simple trisomy 13 and eight cases of translocation trisomy 13. Our results show the efficiency of combining cytogenetic with molecular analysis to study parental origin, as we were able to specify the parent and meiotic stage of origin in all cases studied in this way. We also show that pairing failure is not the most important cause of trisomy involving chromosome 13 , as crossing over between the two non-disjoined chromosomes was detected in almost all cases that were studied.

\section{Materials and methods}

\section{ASCERTAINMENT OF CASES}

From 1976 to 1985 , we conducted a cytogenetic survey of all spontaneous abortions occurring at a single hospital in Honolulu. During the study, we identified 46 abortuses with simple trisomy 13 and 16 abortuses with translocation trisomy 13 , and details of these cases are given in the previous paper in this issue. ${ }^{11}$ Parental blood samples were obtained in 38 cases. In 31 of these, cytogenetic markers alone were used to evaluate the parental origin of the abnormality. In seven cases DNA was obtained from fetal tissue or cultured cells and from

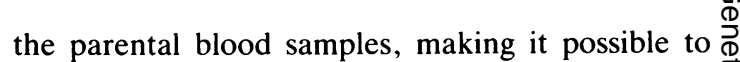
evaluate both cytogenetic and DNA markers.

Three other cases involved liveborn infants, one $\overrightarrow{\vec{s}}$ of whom was a complete trisomy 13 , one a normal/ $\vec{\sigma}$ trisomy 13 mosaic, and one had an unbalanced $13 ; 13$ 듬 translocation. In the latter two cases, the initial $\bar{c}$ cytogenetic diagnosis was made at another labora- $\vec{\otimes}$ tory and the case subsequently referred to us because of our interest in parental origin studies.

CYTOGENETIC ANALYSIS

Chromosome heteromorphisms of the parents and trisomic fetuses or infants were compared using $\mathrm{Q}$ banding with dichloromethoxyacridine/spermine. ${ }^{12}$ All cases were examined directly under the micro- i scope by at least two independent observers, and, in $\vec{N}$ the event of a disagreement, the final decision taken was the most conservative one compatible with both N sets of observations.

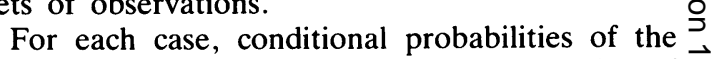
observations were assigned using the formulae of $\vec{\nabla}$ Jacobs and Morton, ${ }^{13}$ and the combined data then $\stackrel{\mathbb{}}{\varnothing}$ used to calculate maximum likelihood estimates of $\frac{\mathbb{S}}{3}$ the different meiotic mechanisms of origin. In all cases, we have assumed that the trisomy resulted from a meiotic and not a mitotic error. Additionallp $\vec{\theta}$ we have assumed that crossing over did not occur between the centromere and the heteromorph region, ${ }^{14}$ and therefore we can specify the meiote stage of origin on the basis of the cytogenetic observations alone.

DNA ANALYSIS

DNA from fetal tissue, cultured fetal cells, and $\overrightarrow{\overrightarrow{0}}$ peripheral blood samples was prepared as pre- 3 viously described. ${ }^{15}$ DNA samples were digested with the appropriate endonucleases using the conditions specified by the manufacturer (International Biotechnologies Inc) and the digested DNA size fractionated overnight on 0.8 to $1.0 \%$ agarose gels. The samples were then transferred to nylon membranes (Zetabind, AMF-Cuno Inc) using the method of Southern ${ }^{16}$ and, after prehybridisation, hybridised to nick translated ${ }^{32} \mathrm{P}$ labelled probes 9 having specific activities of at least $10^{8} \mathrm{cpm} / \mu \mathrm{g}$. $\frac{D}{8}$

Seven probes detecting chromosome 13 RFLPs‥ were used, namely p7F12, p7D2, pHU26, p9D11, N pHUB8, p1E8, and p9A7. Information on the allelic frequencies and fragment sizes, physical locations of the loci defined by the probes, and linkage relation- $\omega$ ships among the loci has recently been summarised by Leppert et al. ${ }^{17}$ Three of the probes (p7D2s? pHU26, and p1E8) detect variation with oneळ enzyme, but the remainder are useful with more? than one enzyme. Thus, by studying p7F12 and p9D11 with TaqI and MspI digested samples, p9A7? 
with HindIII and MspI digested samples, and pHUB8 with EcoRI and HindIII digested samples, a total of 11 probe/enzyme systems was used in the analysis.

\section{Results}

PARENTAL ORIGIN OF TRISOMY

Detailed information on the 33 cases of simple

TABLE 1 Summary of parental origin data from 31 spontaneous abortions and two liveborns with an additional chromosome 13.

\begin{tabular}{|c|c|c|c|c|c|c|c|c|c|}
\hline \multirow[t]{2}{*}{ ID No } & \multirow[t]{2}{*}{$\begin{array}{l}\text { Chromosome } \\
\text { constitution }\end{array}$} & \multicolumn{3}{|c|}{$\begin{array}{l}\text { Chromosome } \\
\text { heteromorphisms }\end{array}$} & \multicolumn{4}{|c|}{$\begin{array}{l}\text { Conditional } \\
\text { probabilities for }\end{array}$} & \multirow[t]{2}{*}{$\begin{array}{l}\text { Parental } \\
\text { origin }\end{array}$} \\
\hline & & Father & Mother & Fetus & $\begin{array}{l}\text { Paternal } \\
\text { I }\end{array}$ & II & $\begin{array}{l}\text { Maternal } \\
I\end{array}$ & II & \\
\hline \multicolumn{10}{|c|}{ Spontaneous abortions } \\
\hline K431 & $47, X Y,+13$ & aa & aa & aaa & 1 & 1 & 1 & 1 & Unknown \\
\hline K435 & $47, X Y,+13$ & $a b$ & $\mathrm{~cd}$ & acd & 0 & 0 & 1 & 0 & Maternal I \\
\hline K662 & $47, X Y,+13$ & ab & cd & acd & 0 & 0 & 1 & 0 & Maternal I \\
\hline K687 & $47, X X,+13$ & $a b$ & cd & aac & 0 & 1 & 0 & 0 & Paternal II \\
\hline K1127 & $47 . X Y,+13$ & $a b$ & cd & $a b c$ & 1 & 0 & 0 & 0 & Paternal I \\
\hline K1154 & $47 . X X+13$ & $a b$ & cd & acc & 0 & 0 & 0 & 1 & Maternal II \\
\hline K1331 & $47, X X .+13$ & ab & $\mathrm{cd}$ & acd & () & () & 1 & 0 & Maternal I \\
\hline K1973 & $47 . \times X .+13$ & $a b$ & aa & aaa & () & $1 / 2$ & $1 / 2$ & $1 / 2$ & Unknown \\
\hline K2015 & $47 . X X .+13$ & $a b$ & $\mathrm{cc}$ & acc & 0 & 0 & 1 & 1 & Maternal I or II \\
\hline K2067 & $47, X X .+13$ & aa & bc & $a b c$ & 0 & 0 & 1 & () & Maternal I \\
\hline $\mathrm{K} 2109$ & $47 . X Y,+13$ & $a b$ & ac & $a b c$ & $1 / 2$ & 0 & $1 / 2$ & 0 & Unknown \\
\hline K2157 & $47 . \times X+13$ & $a b$ & ac & $a b c$ & $1 / 2$ & 0 & $1 / 2$ & 0 & Unknown \\
\hline K2234 & $47 . X Y,+13$ & - & $a b$ & aab & 1 & 1 & 1 & 1 & Unknown \\
\hline $\mathrm{K} 2260$ & $47, X Y,+13$ & $a b$ & ac & aac & 0 & $1 / 4$ & $1 / 2$ & 0 & Unknown \\
\hline K2528 & $47 . X X .+13$ & $a b$ & aa & aab & 1 & 0 & $1 / 2$ & $1 / 2$ & Unknown \\
\hline K2707 & $48 . X Y,+13,+21$ & $a b$ & cd & acd & () & 0 & 1 & 0 & Maternal I \\
\hline K2710 & $47 . X Y,+13$ & $a b$ & cc & acc & 0 & 0 & 1 & 1 & Maternal 1 or II \\
\hline K2722 & $47 . \times X+13$ & $a b$ & ac & $a b c$ & $1 / 2$ & 0 & $1 / 2$ & 0 & Unknown \\
\hline K2766 & $47, X Y,+13$ & $a b$ & $\mathrm{~cd}$ & $a b c$ & 1 & 0 & 0 & 0 & Paternal I \\
\hline K2788 & $47 . \times X .+13$ & aa & $a b$ & aab & $1 / 2$ & $1 / 2$ & 1 & 0 & Unknown \\
\hline K2855 & $47, X Y,+13$ & aa & bb & $a b b$ & 0 & 0 & 1 & 1 & Maternal I or II \\
\hline K2857 & $47 . X X .+13$ & aa & ab & aab & $1 / 2$ & $1 / 2$ & 1 & 0 & Unknown \\
\hline K2858 & $47 . X Y .+13$ & $a b$ & cd & acc & () & 0 & 0 & 1 & Maternal II \\
\hline K3057 & $47 . X X .+13$ & $a b$ & cd & acd & () & () & 1 & 0 & Maternal I \\
\hline K3177 & $47 . \times X .+13$ & aa & $b c$ & $a b c$ & () & 0 & 1 & 0 & Maternal I \\
\hline K3178 & $47 . \times X .+13$ & $a b$ & cd & acd & 0 & () & 1 & 0 & Maternal I \\
\hline K3283 & $47, X X,+13$ & $a b$ & cd & acd & () & () & 1 & () & Maternal I \\
\hline K3325 & $47 . X Y+13$ & aa & bc & $a b c$ & 0 & 0 & 1 & 0 & Maternal 1 \\
\hline \multirow[t]{2}{*}{ K3362 } & $46 . X .+13$ & $a b$ & ac & $\mathbf{a a b}$ & $1 / 2$ & 0 & 0 & $1 / 4$ & Paternal I \\
\hline & & aa & bb & $a a b^{*}$ & 1 & 1 & 0 & 0 & \\
\hline \multirow[t]{2}{*}{ K3392 } & $47 . X Y,+13$ & $a b$ & ac & acc & 0 & 0 & 0 & $1 / 2\}$ & Maternal II \\
\hline & & aa & bb & $a b b^{*}$ & 0 & () & 1 & $1\}$ & \\
\hline G72 & $47, X Y,+13$ & $a b$ & cd & acd & () & 0 & 1 & 0 & Maternal I \\
\hline \multirow[t]{2}{*}{$\times 16$} & $46, X X / 47, X X .+13$ & aa & $a b$ & $\mathrm{bb} / \mathrm{abb}$ & () & 0 & 0 & $1\}$ & Maternal II \\
\hline & & aa & $a b$ & $a b b^{*}$ & () & 0 & 1 & $1\}$ & \\
\hline
\end{tabular}

${ }^{*}$ Results of analysis of DNA polymorphisms.

TABLE 2 Summary of studies of parental origin in 30 cases of trisomy $13^{*}$.

\begin{tabular}{|c|c|c|c|c|c|c|c|}
\hline & \multicolumn{7}{|c|}{ Meiotic origin of non-disjunction } \\
\hline & $\begin{array}{l}\text { Paternal } \\
\text { I }\end{array}$ & II & Either & $\begin{array}{l}\text { Maternal } \\
I\end{array}$ & II & Either & Unknown \\
\hline No of cases & 2 & 1 & 0 & 10 & 4 & 3 & 10 \\
\hline $\begin{array}{l}\text { Maximum likelihood } \\
\text { estimate for } \\
\text { occurrence of errort }\end{array}$ & $0 \cdot 12$ & $0 .(1) 4$ & - & 0.68 & $0 \cdot 16$ & - & - \\
\hline \multirow[t]{2}{*}{$\begin{array}{l}\text { Mean maternal } \\
\text { age (SD) }\end{array}$} & $\begin{array}{l}26 \cdot 5 \\
(12 \cdot())\end{array}$ & 32 & - & $\begin{array}{l}32 \cdot 6 \\
(5 \cdot 7)\end{array}$ & $\begin{array}{l}36 \cdot 5 \\
(7 \cdot 9)\end{array}$ & $\begin{array}{l}29 \cdot 3 \\
(5 \cdot 9)\end{array}$ & $\begin{array}{l}27 \cdot 9 \\
(5 \cdot 6)\end{array}$ \\
\hline & \multicolumn{3}{|c|}{$28 \cdot 3(9 \cdot 1)$} & \multicolumn{3}{|c|}{$32.9(6.3)$} & \\
\hline
\end{tabular}

*Excludes one mosaic trisomy 13 (X16) and two double aneuploids (K2707 and K3362).

†Based on the formulae provided by Jacobs and Morton. ${ }^{13}$ 
trisomy 13 is given in table 1 and a summary of the parental origin studies on the 30 single, complete trisomies given in table 2. Examples of the use of RFLPs in determining parental origin are provided in fig 1 . We were able to determine the origin of the

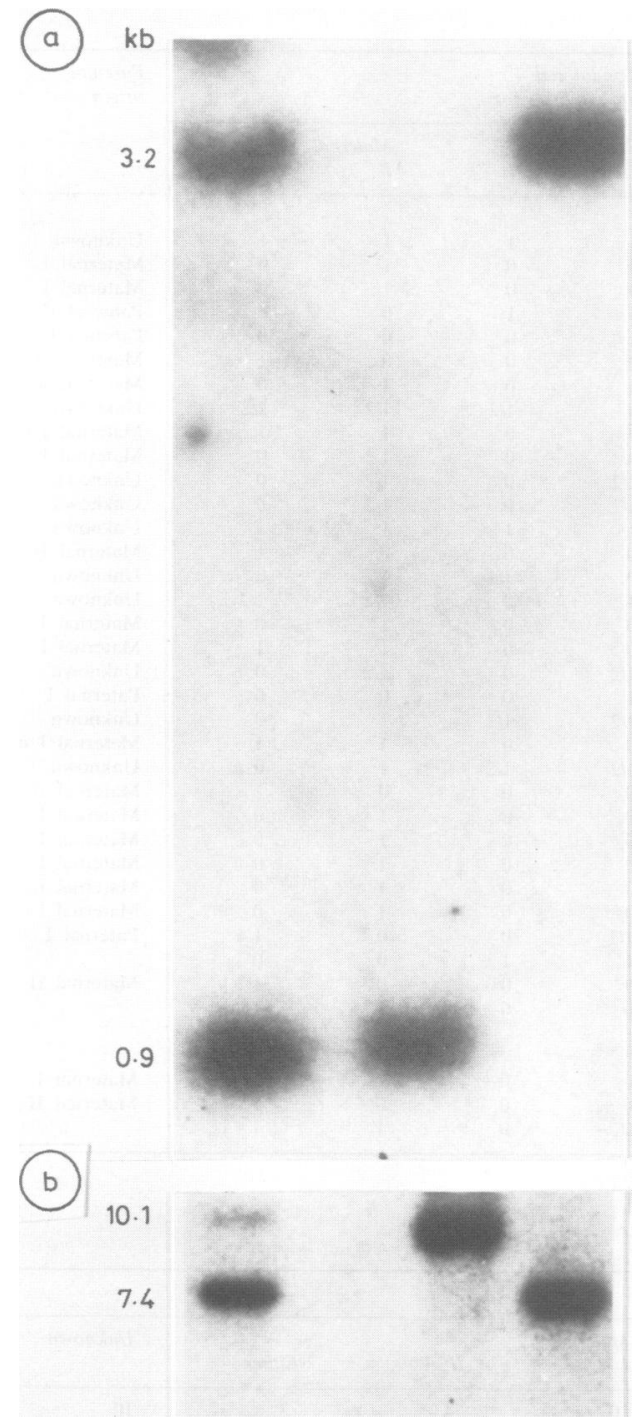

FIG 1 Use of RFLPs to determine the parental origin of trisomy 13 (in both $a$ and $b$, lane $1=$ trisomy, lane $2=$ father, lane $3=$ mother). (a) Paternal origin of trisomy. Samples digested with HindIII and probed with $p 9 A 7$. 1:2 ratio of upper to lower band in this example and in b below is consistent with scanning densitometric readings of normal heterozygotes and trisomies with the opposing genotype. (b) Maternal origin of trisomy. Samples digested with MspI and probed with plE8. additional chromosome in 20 of the 30 cases, with 17 $(85 \%)$ being maternal and three $(15 \cdot 0 \%)$ being paternal in origin. The single most common mechanism of origin was maternal meiosis I non-disjunction, which accounted for an estimated $68 \%$ of cases.

Three cases were considered separately as they $\overparen{\pi}$ had more complicated karyotypes. One of these (X16) was a normal/trisomy mosaic with the normal cell line comprising $64 \cdot 3 \%(45 / 70)$ of all cells $\vec{\circ}$ examined. Cytogenetic analysis showed that, like most mosaic trisomies, ${ }^{18}$ the conception began development as a trisomic zygote, in this case as a result of a maternal meiosis II error. Subsequently the paternal chromosome 13 was lost, presumably at an early division of the zygote, as mosaicism was present in both blood and skin. Thus the 'normal' ? cell line was, in fact, disomic for a maternal N chromosome 13 and had no paternal contribution.

The two remaining cases were both double aneuploids, with K2707 being trisomic for chromosome 21 and $\mathrm{K} 3362$ being monosomic for the $\mathrm{X}$ as well as being trisomic for 13 . In the former case, we were unable to determine the parental origin of the additional chromosome 21, but could not exclude an error at maternal meiosis I (the source of the extra $\bar{\emptyset}$ chromosome 13). In the latter case, analysis of linked RFLPs indicated that the single $X$ was. maternal in origin and, by combining the cytos genetic with the molecular results (table 3), the origin of the additional chromosome 13 was determined to be non-disjunction at paternal meiosis I. Thus, in this instance, both fetal chromosome abnormalities involved the paternal complement.

Table 2 also summarises information on maternal age for trisomy of paternal and maternal origin. Although the data are limited, they indicate a strong maternal age component in cases of maternal meiosis I and II origin, but there is no such evidence for the paternally derived cases. This is consistent with the idea that higher maternal age increases the likelihood of non-disjunction and provides no support for the 'relaxed selection' hypothesis, ${ }^{19}$ which suggests that the association between trisomy $\frac{9}{5}$ and maternal age derives, at least in part, from $\frac{D}{O}$ decreasing ability to abort an abnormal conceptus with increasing maternal age.

The data on maternal age also provide indirect evidence against the 'production line' hypothesis, ${ }^{7} \mathrm{~N}$ which suggests that the effect of maternal age on $\omega$ trisomy results from an increased likelihood of univalency in aged oocytes. If this were the case, $\mathscr{C}$ maternal age should be raised in trisomy of maternal meiosis I, but not meiosis II, origin. In fact, we observed the highest mean maternal age in trisomy of maternal meiosis II origin. This observation is 


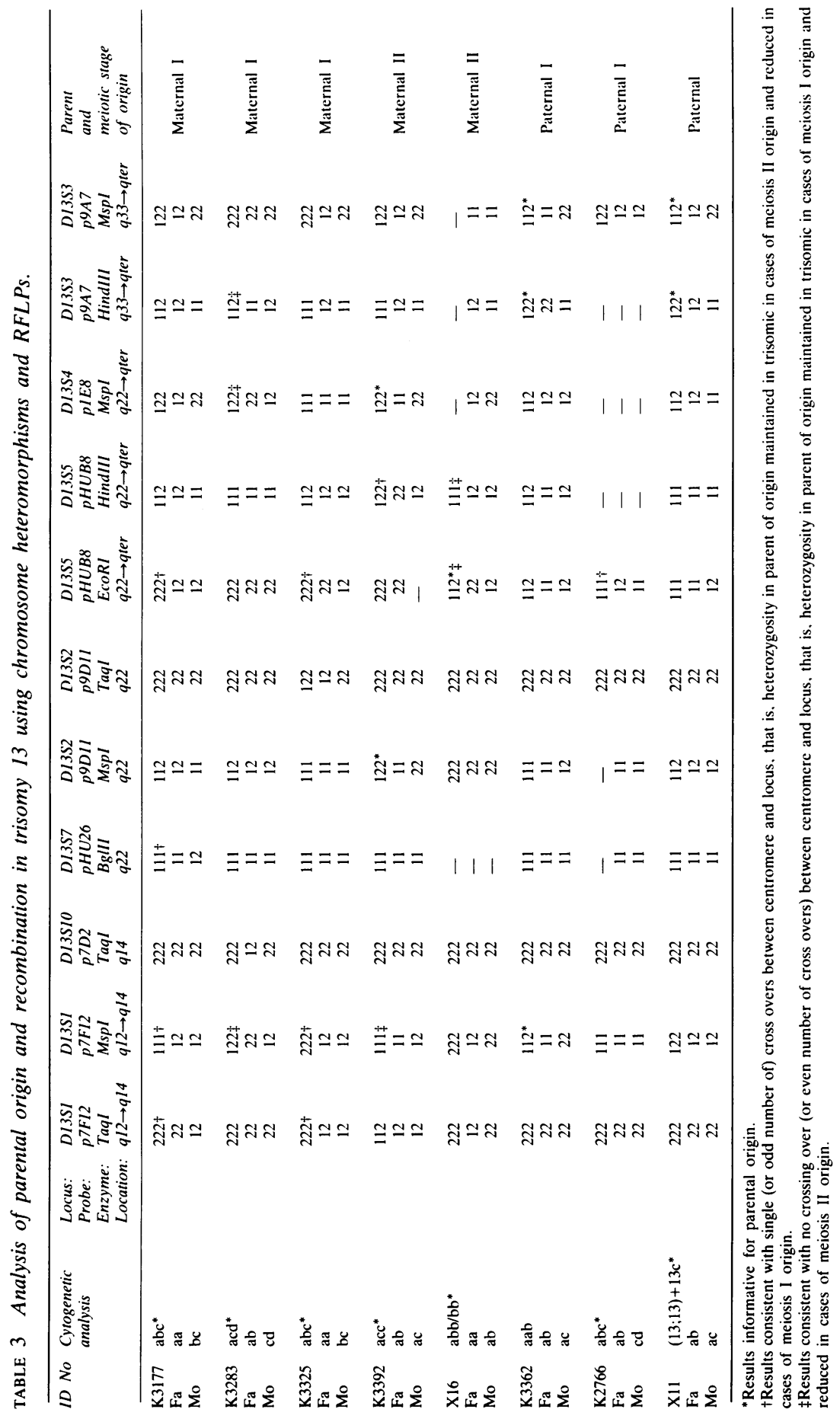


based on a limited number of cases, but it is substantiated by cytogenetic studies of trisomy 21 , which have consistently found the highest maternal ages to be associated with non-disjunction at maternal meiosis II. ${ }^{2}$ Thus, it seems likely that maternal age adversely affects chromosome segregation at both meiotic divisions. As recombination occurs only at the first meiotic division, it is doubtful that a deleterious effect of increasing age on recombination is the mechanism responsible for the dramatic increase in non-disjunction with advancing age. Instead, it seems more likely that ageing affects organelles such as the centromere or the spindle, which are common to both meiotic divisions.

\section{PARENTAL ORIGIN OF TRANSLOCATION}

TRISOMY 13

Table 4 summarises inforration on studies of parental origin for seven fetuses and one liveborn infant with de novo translocation trisomy 13 . We were able to determine the origin of the translocation chromosome in four of the eight cases, three on the basis of cytogenetic evidence and one on the basis of both RFLPs and chromosome heteromorphisms. In two instances the origin of the abnormality was maternal and in two it was paternal. These results are similar to previous reports of de novo Robertsonian translocations, which have found approximately equal frequencies of paternally and maternally derived translocations. ${ }^{20}$

\section{RECOMBINATION AND NON-DISJUNCTION}

Table 3 summarises results on the seven trisomies and one translocation trisomy which were studied using both RFLPs and chromosome heteromorphisms. We were able to determine the parent and meiotic stage of origin of the additional chromosome in all seven trisomies, and therefore in each case were able to study crossing over between the centromere and the loci tested.

Crossing over between the two non-disjoined chromosomes clearly occurred in at least two of three trisomies of maternal meiosis I origin (fig 2).믐 In $\mathrm{K} 3177$, heterozygosity in the mother was reduced? to homozygosity in the fetus at D13S1, D13S7, and D13S5. Similarly, in K3325 heterozygosity waso reduced at $D 13 S 1$ and $D 13 S 5$, with D13S7 being uninformative. Thus, in both trisomies the mosto economical explanation of the results is a single $\overrightarrow{-}$ exchange between the centromere and the most proximal locus $(D 13 S 1)$ with no evidence for furthers. recombination. In the other trisomy of maternato meiosis I origin (K3283) maternal heterozygosity was maintained for all three informative loci (D13S1, D13S4, and D13S3) and therefore we were unable to document an exchange between the two N non-disjoined chromosomes.

We were also able to document crossing over in two of the remaining four trisomies. In K3992, one of the two cases of maternal meiosis II origin,, $\mathbb{Q}$ heterozygosity was reduced to homozygosity ats D13S1, consistent with no crossing over between the centromere and this locus. However, the fetus was heterozygous for the HindIII polymorphism gate $D 13 S 5$, indicating that a cross over had occured between $D 13 S 1$ and D13S5. In K2766, we identifièd a cross over between the centromere and D13S5, thes only locus for which the parental RFLPs were potentially informative. We were unable to identifyo recombinants in the other two cases, but in oneक (X16) only one locus was potentially informative and in the other (K3362) there were no informative loci.

In the translocation trisomy 13 (X11), analysis of both cytogenetic and molecular markers indicated a paternal origin for the abnormality. Additionally,

TABLE 4 Summary of data on parental origin from seven spontaneous abortions and one liveborn infant with de novo translocation trisomy 13.

\begin{tabular}{|c|c|c|c|c|c|}
\hline \multirow[t]{2}{*}{ ID No } & \multirow[t]{2}{*}{ Chromosome constitution } & \multicolumn{3}{|c|}{ Chromosome heteromorphisms } & \multirow{2}{*}{$\begin{array}{l}\text { Parental } \\
\text { origin }\end{array}$} \\
\hline & & Father & Mother & Fetus* & \\
\hline \multicolumn{6}{|c|}{ Spontaneous abortions } \\
\hline K254 & $46, X Y,-14,+t(13 ; 14)$ & $a b$ & aa & $13 / 14+13 b$ & Maternal \\
\hline K340 & $46, X Y,-13,+t(13 ; 13)$ & $a b$ & ac & $13 / 13+13 c$ & Paternal \\
\hline K942 & $46, X Y,-14,+t(13 ; 14)$ & $a b$ & aa & $13 / 14+13 b$ & Maternal \\
\hline K1398 & $46, X Y,-14,+t(13 ; 14)$ & aa & aa & $13 / 14+13 a$ & Unknown \\
\hline K2275 & $46, X X,-13,+t(13 ; 13)$ & $a b$ & $a b$ & $13 / 13+13 a$ & Unknown \\
\hline K2571 & $46, X Y,-14,+t(13 ; 14)$ & $a b$ & $a b$ & $13 / 14+13 a$ & Unknown \\
\hline \multicolumn{6}{|c|}{ Liveborns } \\
\hline $\mathrm{X} 11$ & $46, X X,-13,+t(13 ; 13)$ & aa & $a b$ & $13 / 14+13 b$ & Paternal \\
\hline
\end{tabular}

*Fetus has inherited a translocation chromosome $(13 ; 13$ or $13 ; 14)$ and a normal chromosome, designated 13a, 13b, or 13c. When the parental origin of the normal chromosome is known, it is inferred that the error occurred in the other parent.

tResults of analysis of DNA polymorphisms. 

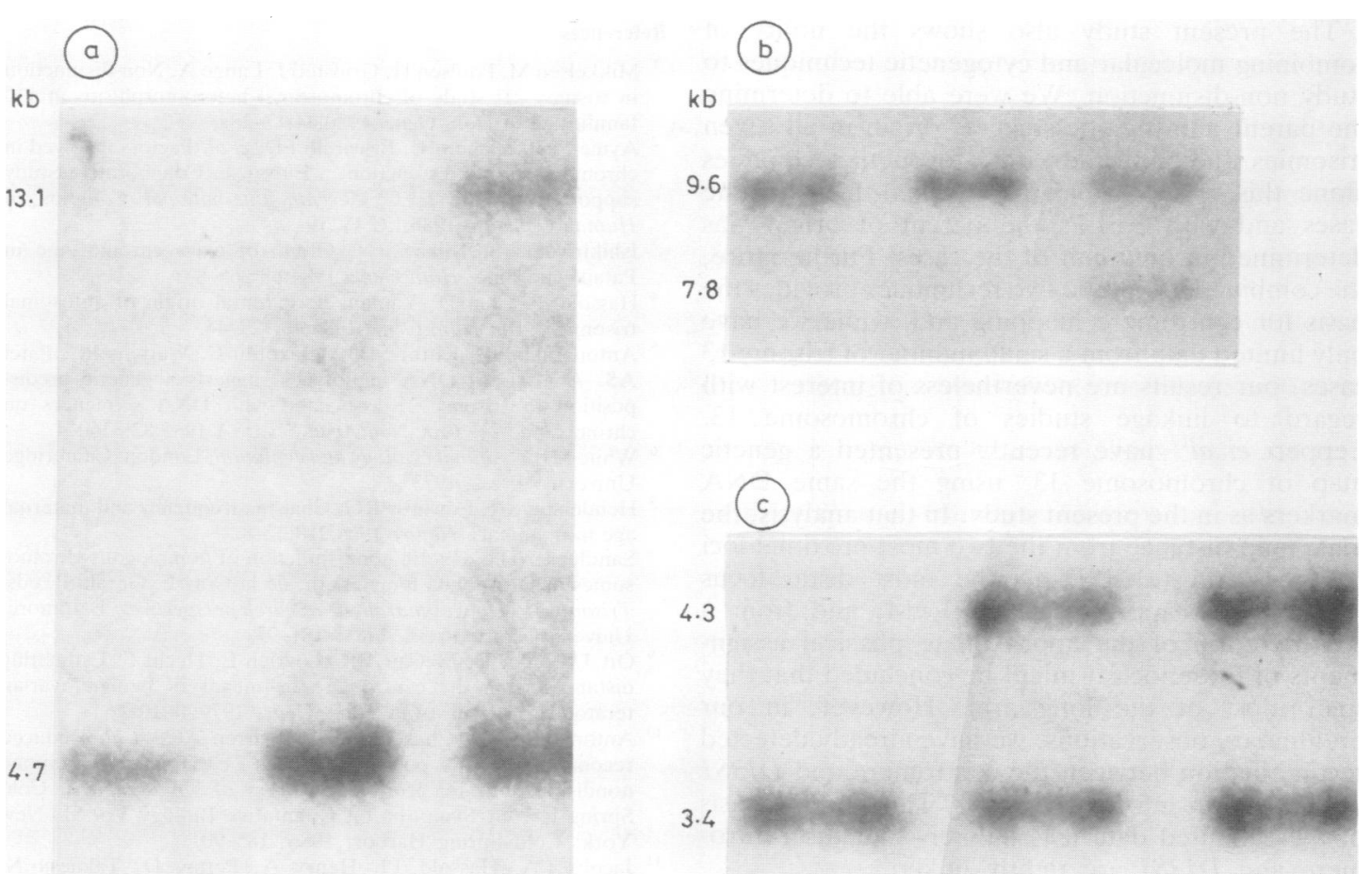

FIG 2 Examples of the use of RFLPs to identify crossing over between the centromere and a marker locus on chromosome 13. In each case, the origin of trisomy was known to be maternal meiosis I and in each case heterozygosity in the mother is reduced to homozygosity in the trisomic offspring (in $a, b$, and $c$, lane $1=$ trisomy, lane $2=$ father, lane $3=m o t h e r$ ). (a) DNA samples digested with EcoRI and probed with pHUB8. (b) DNA samples digested with BgllI and probed with pHU26. (c) DNA samples digested with MspI and probed with $p 7 F 12$.

analysis of RFLPs in the trisomic infant showed that crossing over must have occurred between the two paternal homologues, since at D13S4 paternal heterozygosity was retained and at D13S3 heterozygosity was reduced to homozygosity.

\section{Discussion}

The results of the present study show that nondisjunction at maternal meiosis $I$ is the most common cause of trisomy 13 . This is consistent with observations of a maternal age effect in trisomy $13,{ }^{21}$ with results of previous parental origin studies of trisomy $13,{ }^{3}$ and with similar studies of other autosomal trisomies. ${ }^{4}$ Additionally, the present likelihood estimates of different meiotic errors are remarkably similar to data already available on trisomy 21 , the only other autosomal trisomy that has been extensively studied. Thus, we estimate the proportion of trisomy 13 resulting from paternal meiosis I and II and maternal meiosis I and II to be $0 \cdot 12,0 \cdot 04,0 \cdot 68$, and $0 \cdot 16$ respectively, compared with estimates of $0.13,0.07,0.68$, and 0.13 for trisomy $21 .{ }^{4}$

These estimates agree well with our previous analysis of a smaller series of trisomies, in which we observed a significantly increased proportion of paternal non-disjunction in trisomies 13 and 21 by comparison with all other trisomies. ${ }^{4}$ However, in that report we also considered data on origin obtained from other laboratories and, as the total data set showed no significant difference between trisomy 13 and other trisomies, we suggested that chromosome 21 might be unique in its susceptibility to paternal non-disjunction. That suggestion now seems unlikely to be correct, based on the results of the larger series of trisomy 13 abortuses summarised in the present report. Nevertheless, our analyses continue to support the general idea of variability in frequency of paternal non-disjunction among trisomies, as the estimated level of paternal nondisjunction remains at approximately $5 \%$ for trisomies 22 and 16, the two trisomies for which we have the most information (Hassold and Jacobs, unpublished data). 
The present study also shows the utility of combining molecular and cytogenetic techniques to study non-disjunction. We were able to determine the parent and meiotic stage of origin in all seven trisomies studied, while with cytogenetic techniques alone this was possible in only two-thirds of the cases and with RFLPs the parent of origin was determined in only half of the cases. Furthermore, the combined use of the two techniques provides the basis for centromere mapping and, while we have only limited data from a small number of trisomy 13 cases, our results are nevertheless of interest with regard to linkage studies of chromosome 13 . Leppert et $a l^{17}$ have recently presented a genetic map of chromosome 13, using the same DNA markers as in the present study. In that analysis, the male map distance from the two most proximal loci (D13S6) and (D13S1) to the most distal locus (D13S5) was approximately $70 \mathrm{cM}$, and from a consideration of the approximate physical assignments of these loci, it might be concluded that they span most of the long arm. However, in our preliminary observations, we have already detected recombination between the centromere and D13SI in two of four informative cases. Thus, on the basis of these limited data it is unlikely that the centromere and D13S1 are tightly linked.

Additionally, our results are of considerable interest in view of the purported association between reduced recombination in maternal meiosis I and trisomy $21 .^{10}$ Specifically, we have evidence for recombination in two of the three cases in which the extra chromosome originated in maternal meiosis I, as well as in the single paternal meiosis I case where information on recombination was obtained. These are preliminary observations but, nevertheless, they clearly show that failure to pair is not the only mechanism responsible for nondisjunction in man. However, it is still possible that a proportion of the cases are the result of asynapsis (for example, in K3283 recombinants were not observed in three opportunities), or that a proportion are related to decreased or increased levels of recombination. A much more extensive series of cases, including trisomies with different parental and meiotic origins and involving parents in different age categories, must be examined before the possible role of recombination in the genesis of human trisomy is fully understood.

We wish to express our gratitude to Drs Jessica Davis and Meira Shaham for providing samples from liveborn subjects with trisomy 13 and their parents and to Norma Takaesu for providing expert technical assistance. This work was supported by NIH grants HD21341 and BRSG S07 RRO5396.

\section{References}

1 Mikkelsen M, Poulsen H, Grinsted J, Lange A. Non-disjunctiơ in trisomy 21: study of chromosomal heteromorphisms in 1. families. Ann Hum Genet 1980;44:17-28.

2 Aymé S, Baccichetti C, Bricarelli FD, et al. Factors involved chromosomal nondisjunction: a European Collaborative Stu supported by the EEC. Seventh International Congress कू Human Genetics, 1986:I:C IV.19.

${ }^{3}$ Ishikiriyama S, Niikawa N. Origin of extra chromosome 잉 Patau syndrome. Hum Genet 1984;68:266-8.

${ }^{4}$ Hassold T, Chiu D, Yamane J. Parental origin of autosomat trisomies. Ann Hum Genet 1984:48:129-44.

5 Antonarakis SE, Kittur SD, Metaxotou C, Watkins PC, Patet AS. Analysis of DNA haplotypes suggests a genetic predfo position to trisomy 21 associated with DNA sequences of chromosome 21. Proc Natl Acad Sci USA 1985;82:3360-4.

${ }^{6}$ White MJD. Animal cytology and evolution. London: Cambridg University Press, 1973.

${ }^{7}$ Henderson SA, Edwards RG. Chiasma frequency and maternal age in mammals. Nature 1968;218:22-8.

* Sandler L. The meiotic nondisjunction of homologous chromid somes in Drosophila females. In: de la Cruz F, Gerald P, eds Trisomy 21 (Down syndrome): research perspectives. Baltimoro: University Park Press, 1981: 181-97.

${ }^{9}$ Ott J, Linder D, McCaw BK, Lovrien E, Hecht F. Estimating distances from the centromere by means of benign ovariof teratomas in man. Ann Hum Genet 1976;40:191-6.

10 Antonarakis SE, Chakravarti A, Warren AC, et al. Reduce recombination rate on chromosomes 21 that have undergo nondisjunction. In: Molecular biology of homo sapiens. Cofe Spring Harbor Symposia on Quantative Biology Vol 51. New

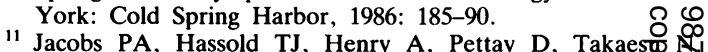
Trisomy 13 ascertained in a survey of spontaneous abortmps $J$ Med Genet 1987;24:721-4.

12 Deugau JH, van de Sande JH, Lin CC. (CMA) $)_{2} \mathrm{~S}$ : a dợb intercalating analogue of quinacrine for chromosome bandin Am J Hum Genet 1978;30:78A.

13 Jacobs PA, Morton NE. Origin of human trisomies añ polyploids. Hum Hered 1977;27:59-72.

14 Lauric DA. Hultén MA. Further studies on bivalent chiasnat frequency in human males with normal karyotypes. Ann $\mathrm{Hu}$ Genet 1985;49:189-201.

15 Hassold T, Kumlin E, Leppert M, Takaesu N. Determinati鱼 of the parental origin of sex chromosome monosomy using restriction fragment length polymorphisms. Am J Hum Gene: 1985;37:965-72.

${ }^{16}$ Southern EM. Detection of specific sequences among DNA fragments separated by gel electrophoresis. J Mol Biol 1975;98 503-17.

17 Leppert M, Cavenee W, Callahan P, et al. A primary gencige map of chromosome 13q. Am J Hum Genet 1986:98:425-3

${ }^{18}$ Hassold T. Mosaic trisomies in human spontaneous abortions. Hum Genet 1982;61:31-5.

19 Aymé S, Lippman-Hand A. Maternal age effect in aneuploidy. does altered embryonic selection play a role? Am J Hum Gene 1982;34:558-65.

${ }^{20}$ Chamberlin J, Magenis RE. Parental origin of de nong chromosome rearrangements. Hum Genet 1980;53:343-7.

${ }^{21}$ Risch N, Kline J, Stein Z, Warburton D. Maternal a dependent and independent factors in autosomal trisomies. $A$ po J Hum Genet 1986;39:68-79.

Correspondence and requests for reprints to $\operatorname{Dr} P$ 勇 Jacobs, Division of Human Genetics, Department of Pediatrics, Cornell University Medical College 515 East 71st Street, New York, NY 10021, USA 